\title{
The challenge of leading with multiple and severe gastrointestinal complications in a patient with systemic sclerosis
}

\author{
Raquel Batista, Lara Adelino, Cristina Lopes, Maria de Fátima Pimenta \\ Serviço de Medicina Interna, Centro Hospitalar Médio Tejo, Abrantes
}

\begin{abstract}
Introduction
Systemic sclerosis (SSc) is a rare chronic autoimunne-mediated connective tissue disorder. Although the aetiology of the disease remains undetermined, systemic sclerosis is characterized by fibrosis and proliferative vascular lesions of the skin and internal organs. Prevalence of the disease in Europe and USA is estimated at 8-30 per 100.000 people with an annual incidence of 1-2 per 100.000 people 1 .

Involvement of the gastrointestinal tract can be found at any form of SSc, but is more frequent in patients with diffuse disease ${ }^{2}$. Gastrointestinal symptoms are seen in 90\% of all the patients with SSc, and are the presenting feature of disease in $10 \%$ of the individuals. The involvement can extend from the mouth to the anus, with the oesophagus and anorectum most frequently affected. Gastrointestinal involvement is the leading cause of morbidity and the third most commmon cause of mortality in patients with systemic sclerosis, with cardiopulmonary and renal involvement
\end{abstract}

being the first and second most common causes, respectively ${ }^{3}$. Severity of gastrointestinal disease has been shown to be a marker of worse prognosis and mortality in patients with systemic sclerosis ${ }^{4,5}$. Gastrointestinal disease can be present with esophageal dysmotility, gastroparesis, small bacterial intestinal overgrowth, colonic dysmotility and fecal incontinence. The symtoms have a profound impact on quality of life. Severe gastrointestinal disease is associated with pseudo-obstruction and malabsorption and may result in dependence on enteral or total parenteral nutrition ${ }^{6}$. Given the heterogenicity of symptoms and the complexitie related to the management, these patients become routinely a challenge.

Keywords: Systemic sclerosis. Gastrointestinal tract. Dismotility. Malabsorption.

Palabras clave: Esclerosis sistémica. Tracto gastrointestinal. Dismotilidad. Malabsorción.

\section{Case report}

A sixty seven year old female caucasian patient, was admitted to the emergency room for investigation of chronic diarrhea without blood, mucus or pus accompanied by ocasional episodes of vomiting with about six month of evolution, that had been worsening in the last days. Her previous medical history included the diagnosis of difuse systemic sclerosis diagnosed three years ago with pulmonary and cardiac involvement, manifestated by intersticial pulmonary fibrosis, cardiac insuficiency with depressed left ventricule contractility and atrial fibrillation. She was medicated with azathioprine and prednisolone $5 \mathrm{mg} /$ day, esomeprazole, calcium, bisoprolol and dabigatran.

At the admission the patient was thin $\left(\mathrm{BMl}=17.9 \mathrm{~kg} / \mathrm{m}^{2}\right)$, presented microstomia, difuse skin thickening of all the body, Raynaud phenomenon, signs of dehydration, abdominal distension, with no abdominal pain on observation. Laboratory tests showed anemia of chronic disease (hemoglobin $=10.6 \mathrm{~g} / \mathrm{dl}$, ferritin 266 ug/L, serum iron $21 \mathrm{ug} / \mathrm{dL})$, hypoalbuminemia $(1.6 \mathrm{mg} / \mathrm{Dl})$ and hyponatremia of $131 \mathrm{mmol} / \mathrm{L}$. Calcium, acid folat,vitamines $\mathrm{D}$ and B12 were normal. Stool tests were performed with the presence of many fat droplets. The abdominal $\mathrm{x}$-ray showed some distended small- bowel loops and air- fluid levels. A computarized tomography was performed and showed little pleural and pericardium effusion, ascites and distension of the small-bowel with no mechanical obstacle. The patient was hospitalized for clinical compensation with the probable diagnosis of small intestinal bacterial overgrowth and intestinal pseudo-obstruction, started endovenous hidratation and antimicrobial therapy with metronidazole.

During the first two weeks of hospital admission the patient got worse with increase of the vomiting episodes, abdominal disten- sion with pain and obstipation in spite of the use of prokinetic and antiemetic therapy. So it was decided to entubate the patient with a nasogastric in order to drain gastric stasis content for abdominal descompression. Unfortunately there was litle clinical improvement. In order to better evaluation of the small intestine a MRI enterography was performed and showed significant frank dilatation jejunal ileal diffuse, still maintaining mucous pattern evident jejunal level but with diffuse erasure of the mucosal relief and with colic also distended and water content more notoriously in the right segments and in smaller volumetric amplitude of the left segment (Fig. 1 and 2). Therefore, and after multidisciplinary discussion, it was decided to start rifaxamine,subcutaneous octreotide, and intravenous immunoglobulin therapy. The patient got clinically better for some weeks tolerating oral feeding, but maintaining abdominal disconfort and intermitent diarrhea. Once the significant malnutrition persisted with an unsucessful enteral feeding at day 40 of hospitalization it was started parenteral feeding. At this time the complaints of pirose, worsening of vomiting and sensation of dysphagia were the main issues. We decided to undertake na endoscopy, that showed extensive plaques not detachable and very suggestive of esophageal candidiasis with distal third spared with the performing of biopsies. Beyond this endovenous fluconazol was started with some benefit. Unfortunatly, after 64 days of hospitalization and clinical improvement of the gastrointestinal manifestations the patient had a severe urosepsis to Klebsiella pneumonie and died.

\section{Discussion}

Whatever the aetiology of the disease, vascular instability is thought to progress to tissue ischaemia, and subsequently 
Figure 1. Coronal MRI enterography image of jejunal-ileal and colonic dilatation.

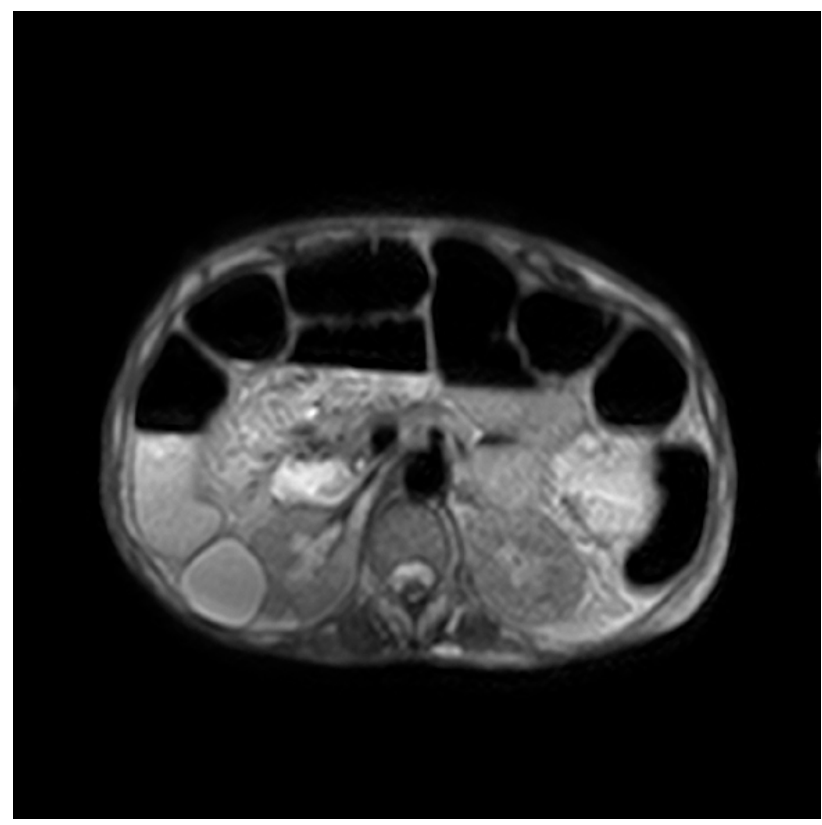

alter mucosal immunity and tissue repair. Tissue repair in these patients is characterized by excessive collagen deposition, resulting in fibrotic replacement of the tissue in the skin and internal organs, including the gastrointestinal tract ${ }^{7}$. Gut involvement can progress rapidly, within few years, in diffuse systemic sclerosis, as presented in this case or slowly within decades in case of limited systemic sclerosis.

Delayed gastric empyting is seen in $50 \%$ of patients with systemic sclerosis ${ }^{8}$. The functional delay seems to relate to both estrutural changes and slowing of gastric electrical activity associated with autonomic nerve. The typical gastroparesis symptoms of abdominal bloating, pain, early saciety and vomiting are more commonly reported in diffuse SSc. Disappointing is the lack of efficacy of prokinetic and entiemetic drugs in these patients, once they probably have no effect in advanced SSc when there is already a smooth muscle atrophy ${ }^{7}$. This patient tried different prokinetic and entiemetic drugs with no clinical improvement. Although a consensus guideline on the management of gastroparesis in general suggests the use of the liquid formulation of metoclopramide, there is no specific evidence for gastroparesis in SSc9.

The small bowel dismotility may be seen up to 40 to $90 \%{ }^{10}$ of the patients and it is associated with small intestine bacterial overgrowth (SIBO) and pseudo-obstrution. The SIBO often presents with prandial distension, nausea, diarrhea and excessive flautulence. Associated with malabsorption of vitamin B12, iron and fat soluble vitamins. The criterion standard for diagnosis is endoscopy with aspiration of proximal jejunal contents with growth of more than 100, 000 colony- forming units per millimeter ${ }^{11}$. The role of jejunal aspiration, an invasive procedure has been largely replaced with the hydrogen breath test ${ }^{7}$. As this test is limited, the alternative is to test SIBO with empirical treatment with broad-spectrum (against
Figure 2. Axial MRI enterography image of jejunal-ileal and colonic dilatation.

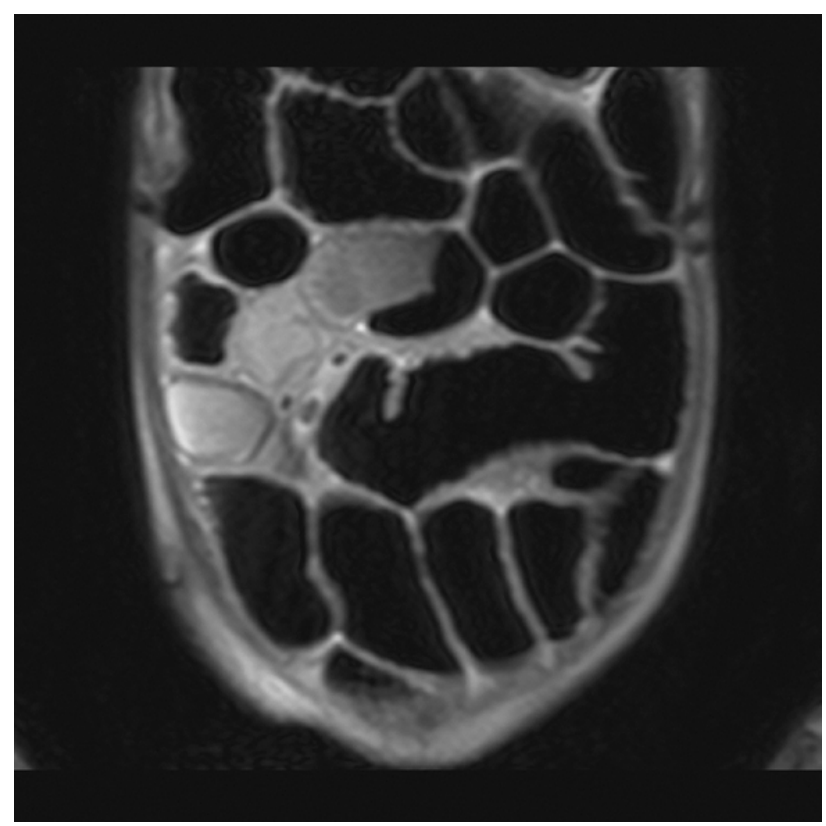

both aerobic and anaerobic strains) to modify the microbiota sufficiently to result in symptomatic improvement, rather than attemping to erradicate the specific strain. These antibiotics include rifaximin, ciprofloxacin and metronidazole, among others ${ }^{7}$. Rifaxamin in particular and which we started in our patient, is an attractive option as it does not confer a risk for Clostridium difficile infection and has a minimal intestinal absorption ${ }^{12}$. The general approach is to treat with one antibiotic for 10-14 days. If symptoms return soon after therapy, a rotating course of antibiotics can be used, where patients are treated for 10-14 days with one antibiotic in one month and then another in the next month, and vice versa ${ }^{12}$. Small intestinal dysmotility leading to stasis of intestinal contents and dilation in the absence of a mechanical lesion is referred to as pseudo-obstrution. As no mechanical lesion exists, a critical goal of treatment is to avoid surgery, which by definition cannot be curative. First line therapy for acute pseudo-obstrution is to rehydrate the patient, estabilish analgesia (preferably avoiding opiods that tend to exacerbate intestinal dymotility), correction of electrolyte abnormalities and decompress the bowel by nasogastric intubation? ${ }^{7}$. Sucutaneous octreodite, a somastatin analogue, has proved beneficial in several series of patients with SSc with intestinal pseudo-obstrution in terms of improving gut motility and alleviating symptoms ${ }^{13}$. Dosing commences at 50 ug twice a day and can be increased to a maximum of 200 ug per day according to treatment response. In this case the right colon was also committed supporting the extensive gastrointestinal involvement of the case.

Beyond all the gastrointestinal involvement described, the patient was also presented with a candida esophagitis, which occurs as an opportunistic infection in immunocompromised hosts. In these patients besides treatment with immu- 
nosuppressive therapy it is also due to the chronic use of proton pump inhibitors and local oesophageal stasis ${ }^{10}$.

Malnutrition in patients with SSc is usually multifactorial in origin. Oral food intake is often reduced as result of persistent and debilitating symtoms secondary to gut dismotility and to SIBO that causes maldigestion and malabsorption of specific nutrients. Due to the insidious onset of the disease and delay of recognition, the problems with nutrition are often present for many months and years ${ }^{14}$. If the patients remain malnourished or symptomatic despite medical therapies then it might need to be considered enteral nutrition. If enteral feeding is not praticable or successful and malnutrition continues, consideration of parenteral feeding is appropriate.

Once we were upon a complex case of an advanced difuse SSc with multiple and severe gastrointestinal complications refractory to all the measures taken, it was started intravenous immunoglobulin therapy. An observational study with SSc patients with refractory inflamatory myopathy showed clinical improvement in various clinical aspects, including gastrointestinal disease. Patients reported improvement in gastrointestinal symptoms, suggesting a possible benefit in a subset of SSc patients with severe and dibilitating gastrointestinal disease and which was also reported in this patient ${ }^{15}$. This case emphasizes the importance of early and continuous monitoring,via regular clinical assessement of gastrointestinal tract involvement in patients with systemic sclerosis in order to avoid the severe syndromes. Small bowel dismotility is a difficult task challenging the clinician in many ways. In this case the significant involving of the gastrointestinal tract was indirectly responsible for the death of the patient.

\section{Bibliography}

1. chifflot H, Fautrel B, Sordet C, Chatelus E, Sibilia J. Incidence and prevalence of systemic sclerosis : a systematic literature review. Semin Arthritis Rheum. 2004 43: 596-02.

2. Attar A. Digestive manifestations in systemic sclerosis. Ann Med Interne. 2002. 153: 260-4.

3. Hunzelmann N, Genth E, Krieg T, Lehmacher W, Melchers I, Meurer M, et al. The registrary of the German Network for Systemic Scleroderma: frequency of diasease subsets and patterns of organ involvement. Rheumatology (Oxford). 2008. 47:1185-92.

4. Al-Dhaler FF. Pope JE, Quimet JM. Determinants of morbility and mortality of systemic sclerosis in Canada. Semin Arthritis Rheum. 2010; 39:269-77.

5. Mayes MD, Lacey JV Jr, Beebe- Dimmer J, Gillespie BW, Cooper B, Laing TJ, et al. Prevalence, incidence, survival and disease characteristics of systemic sclerosis in a large US population. Arthritis Rheum. 2003; 48:2246-55.

6. Steen VD, Medsgar TA Jr. Severe organ involvement in systemic sclerosis with diffuse scleroderma. Arthritis Rheum. 2000; 43:2437-44.

7. Emmanuel A. Current management of the gastrointestinal complications of systemic sclerosis. Nat Rev Gastroenterol Hepatol. 2016; 13: 461-71.

8. Madsen JL, Hendel L. Gastrointestinal transit times of radiolabeled mal in progressive systemic sclerosis. Dig Dis Sci. 1992; 37:1404-08.

9. Verne GN, Eaker EY, Hardy E, Sninsky CA. Effect of octreotide and erythromycin on idiopathic and scleroderma- associated intestinal pseudoobstruction. Dig Dis Sci. 1995; 40: 1892-901.

10. Savarino E, Mei F, Parodi A, Ghio M, Furnari M, Gentile A, et tal. Gastrointestinal motility disorder asseement in systemic sclerosis. Rheumatology (Oxford). 2013; $52: 1095-100$

11. Kirby DF, Chatterjee $S$. Evaluation and management of gastrointestinal manifestations in scleroderma. Curr Opin Rheumatol. 2014; 26: 621- 9 .

12. Shreiner AB, Murray C, Denton C, Khanna D. Gastrointestinal manifestations of systemic sclerosis. J Scleroderma Relat Disord. 2016; 1: 247-56.

13. Nikou GC, Effect of octreotide on intestinal motility and bacterial overgrowth in scleroderma. N Engl J Med. 1991. 325: 1461-67.

14. Forbes $A$, Marie I. Gastrointestinal complications: the most frequent internal complications of systemic sclerosis. Rheumatology (Oxford). 2009; 48:36-9.

15. Raja J, Nithyanova SI, Murray CD, Denton CP, Ong VH. Sustained benefit from intravenous immunoglobulin therapy for gastrointestinal involvement in systemic sclerosis. Rheumatology (Oxford). 2016; 55: 115-9. 